\title{
HF Source Geolocation using an Operational TDoA Receiver Network: Experimental Results
}

\author{
Ankit Jain, Pascal Pagani, Rolland Fleury, Michel M. Ney and Patrice Pajusco
}

\begin{abstract}
Determining the geographic location of high frequency (HF) transmitters is of prime importance in both defense and civil operations. In this letter, we present experimental results of distant $\mathrm{HF}$ transmitter geolocation performed using the time difference of arrival (TDOA) method. The HF signals considered for our study are transmitted from different locations across Europe. Multiple receivers capable of capturing HF radio signals synchronously are built and a nationwide receiver network with centralized control is deployed in France to assess the feasibility of our approach. HF radio signals are captured simultaneously by all receivers in the network from different European HF broadcast transmitters. From the captured data, the propagation duration differences between the signals received by all receivers are obtained using the cross-channel sounding method. Finally, the transmitter location is estimated using the geolocation algorithm based on the TDoA method. Experimental results demonstrate that it is possible to locate the HF transmitters under favorable conditions with a relative geolocation error ranging from about 0.1 to $10 \%$ of the actual ground distance, for most of the experimental scenarios.
\end{abstract}

Index Terms-Geolocation, HF propagation, Ionosphere, TDoA

\section{INTRODUCTION}

Radio signals from the HF band cover large geographical areas and can be used for long distance communication across intercontinental distances using light and portable devices. Thus, HF communication is extremely useful for defense and civil operations in remote areas. In this framework, the location of HF transmitters can be passively retrieved by capturing and analyzing the HF communication signals. HF radio signals propagate through the ionospheric channel using skywaves; reflected once or multiple times between the Earth and the ionosphere. The ionosphere is composed of neutral atoms which are ionized by the sun's radiation entering the Earth's atmosphere. This results in free electrons which are responsible for the refraction of $\mathrm{HF}$ radio signals. The ionosphere is further split into different layers with varying electron density and the characteristics of these layers are highly variable depending on the solar activity, geographic location and season [1].

Traditionally, HF geolocation was performed by measuring the Direction of Arrival (DoA) of the incoming signals. Different forms of HF direction finding (DF) systems capable of measuring the DoA are explained in [2].

Manuscript received March 14, 2018. This work was supported in part by the Carnot Institute and the Brittany region in France. The authors thank the Atmospheric Optics Laboratory in University of Lille 1 and the Electrical Engineering Department in IUT1-University Grenoble Alps for hosting our receiver setup.

The authors are with the Microwave Department, IMT Atlantique, LabSTICC UMR CNRS 6285, Brest, France (Corresponding author e-mail: ankit.jain@imt-atlantique.fr).
To further improve the accuracy of measured angle of arrival (AoA), several high resolution DoA estimation algorithms such as MUSIC [3] and ESPRIT [4] can be used to resolve the uncorrelated signals. These algorithms face problems when uncorrelated and coherent signals exist together. An efficient DoA method is detailed in [5] which estimates the DoA for both uncorrelated and coherent signals separately. HF radio DF using MUSIC algorithm is experimentally validated by deploying a receiver system based on a heterogeneous circular array [6]. There is a slight bias between the estimated azimuth and elevation angles when compared with the expected values.

Another HF geolocation technique, known as single site location (SSL) estimates the DoA in terms of azimuth and elevation angle of the incoming signal [7]. Moreover using the SSL method, it is possible to locate a HF transmitter located about $2000 \mathrm{~km}$ away from the receiving system with an error of about $10 \%$ [8]. Both SSL method and traditional DF systems require the deployment of large antenna arrays at single or multiple sites which can be quite expensive and bulky. It is also mandatory to have information about the ionospheric propagation path to estimate the ionospheric reflection height.

A study for passive location systems using TDoA method for Line-of-Sight (LOS) transmissions is explained briefly in [9]. In the case of HF geolocation, signals are received through non-line-of-sight (NLOS) mode. HF geolocation is possible using both time of arrival (ToA) and time difference of arrival (TDoA). In our earlier work, it is shown that the location of HF transmitters can be found using the ToA method without any information about the ionospheric profile along the propagation path [10]. Furthermore, single antenna portable receiver systems can be used at multiple sites which makes it more cost-effective compared to a SSL or DF system. When the transmission time of the signal is known, $\mathrm{HF}$ geolocation can be performed using the ToA principle whereas when the transmission time of the signal is unknown; HF geolocation can be performed using the TDoA principle.

In the context of passive geolocation, the transmit time of the signal is not known. Thus, we use the TDoA method to evaluate the geographic location of the HF transmitter. This letter presents for the first time experimental results of HF geolocation using the TDoA method, obtained by collecting data from a country wide receiver network capable of capturing HF radio signals synchronously. In Section II, HF propagation and the geolocation method based on TDoA are described. Section III introduces the HF geolocation system allowing the capture of HF radio signals. In Section IV, experimental geolocation results of different HF transmitters are presented and discussed. In Section V, conclusions are drawn and future work is described. 


\section{HF PROPAGATION AND GEOLOCATION PRINCIPLE}

HF radio signals propagating using skywaves reach the destination via a single or multiple reflections from any ionospheric layer. Signals which are reflected once from the ionosphere are referred to as one-hop propagation mode. Using one-hop mode, communication is possible over distances in the range of 500-3000 km.

The geometry of HF propagation paths, assuming a single reflection from a uniform ionosphere, can be seen in Fig. 1. Moreover, the Earth is assumed to be flat by neglecting its curvature. The transmitting site is located at $\mathrm{Tx}$ while the receiving sites are located at $\mathrm{Rx}_{1}$ and $\mathrm{Rx}_{2}$. Due to the gradient-index in the ionosphere, the HF radio signals travel in a curved path. According to Breit and Tuve's theorem [1], the group path between $\mathrm{Rx}_{1}-\mathrm{Tx}$ indicated by the red curve is equal to the triangular path $\mathrm{Rx}_{1}-\mathrm{I}_{1}-\mathrm{Tx}$ in vacuum; the same applies for $\mathrm{Rx}_{2}-\mathrm{Tx}$. Under the assumption of uniform ionosphere, the actual ionospheric reflection height for both propagation paths is the same and denoted by $h_{m}$. This reflection height is usually obtained from some ionospheric modelling software or an ionosonde. Similarly, the reflection heights for both triangular paths are also equivalent and denoted by $h_{v}$.

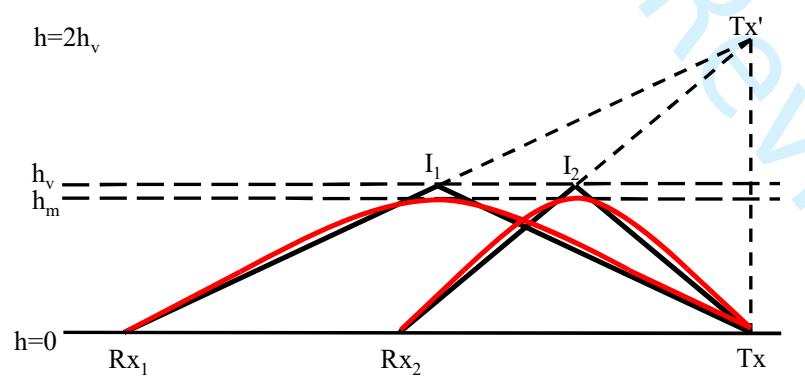

Fig. 1 Geometry of HF geolocation in TDoA [11]

From the electromagnetic image theory, the triangular path length $\mathrm{Rx}_{1}-\mathrm{I}_{1}-\mathrm{Tx}$ is equal to the path length $\mathrm{Rx}_{1}-\mathrm{Tx}^{\prime}$ and the same applies for the second triangular path. Using this geometry, the location of the HF transmitter can be obtained by finding the coordinates of point $\mathrm{Tx}^{\prime}$ without any knowledge of the actual ionospheric reflection height. The group path range differences are obtained by multiplying the propagation duration differences with the speed of light from at least three different receiver pairs. From the known receiver locations and the group path range differences, the point $\mathrm{Tx}^{\prime}$ is found by multilateration. In our previous work we have presented the mathematical approach and the formulae for obtaining the location of Tx' from the TDoA method [11].

\section{HF GEOLOCATION SYSTEM}

Four receivers capable of capturing HF signals synchronously were built using software defined radio (SDR) in order to perform HF geolocation using TDoA method. We installed these receivers in 4 cities in France namely, $\mathrm{Rx}_{1}$ in Brest, $\mathrm{Rx}_{2}$ in Bordeaux, $\mathrm{Rx}_{3}$ in Grenoble, and $\mathrm{Rx}_{4}$ in Lille. The HF receiver prototype is explained in detail in [12]. HF radio signals are captured synchronously by four receivers from five different HF broadcast transmitters located in
Nauen-Germany, Santa Maria di Galeria (SMDG)-Italy, Cerrik-Albania, Galbeni-Romania and Moosbrunn-Austria. The geographic location of all these transmitters and the deployed receivers is presented in Fig. 2. Table 1 provides the ground distances between different HF broadcast transmitters and receivers. Radio signals transmitted using $\mathrm{HF}$ broadcasters are amplitude modulated and transmitted over a $10-\mathrm{kHz}$ band.

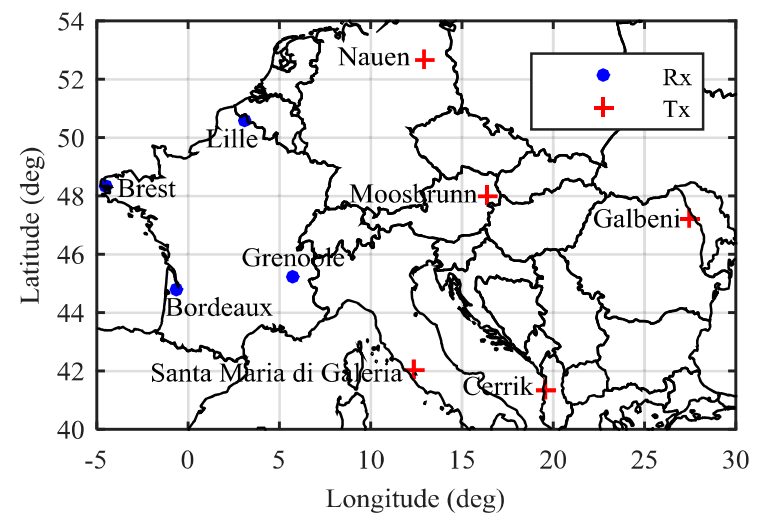

Fig. 2 GPS coordinates of HF receivers and transmitter sites

The automatically captured data files are post-processed and data is retrieved in the form of complex samples (IQ data). To remove other interfering signals which are captured along with the signal of interest, the captured data are filtered in the frequency domain. Over the available $200 \mathrm{kHz}$ band, only 5 $\mathrm{kHz}$ of signal on both sides of the carrier frequency of the transmitter is passed and the rest is filtered out. The signal is transformed back to the time domain and the received signal's average power is calculated. The transformed signal is demodulated to obtain the message signal.

The geolocation algorithm used to find the Tx location is dependent on the reference receiver. Thus, the receiver with the highest signal average power is considered as the reference receiver. The message signal from the reference receiver is cross-correlated with the obtained message signals from the other receivers to obtain the propagation duration differences. The TDoA $(\Delta \mathrm{t})$ corresponds to the maximum value of the cross-correlation function and is given as follows:

$$
\Delta t=\max _{t} R_{s_{r e f} s_{\mathrm{i}}}(t)=\max _{t} \int_{-\infty}^{\infty} s_{\text {ref }}(\tau) s_{\mathrm{i}}(t+\tau) d \tau
$$

where $R_{s_{r e f} s_{i}}$ is the cross-correlation between the message signal received on the reference receiver denoted by $s_{\text {ref }}(t)$ and other receivers denoted by $s_{\mathrm{i}}(t)$, respectively. Finally, the Tx location is estimated using the obtained TDoA values by solving a system based on quadratic equations [11].

Table 1 Great circle distance between the receivers and HF transmitters

\begin{tabular}{|c|c|c|c|c|}
\hline $\mathrm{Tx}$ & $\begin{array}{c}\mathrm{Tx}-\mathrm{Rx}_{1} \\
(\mathrm{~km})\end{array}$ & $\begin{array}{c}\mathrm{Tx}-\mathrm{Rx}_{2} \\
(\mathrm{~km})\end{array}$ & $\begin{array}{c}\mathrm{Tx}-\mathrm{Rx}_{3} \\
(\mathrm{~km})\end{array}$ & $\begin{array}{c}\mathrm{Tx}-\mathrm{Rx}_{4} \\
(\mathrm{~km})\end{array}$ \\
\hline Nauen & 1320 & 1313 & 976 & 710 \\
\hline SMDG & 1493 & 1085 & 632 & 1182 \\
\hline Cerrik & 2049 & 1680 & 1197 & 1631 \\
\hline Galbeni & 2376 & 2168 & 1676 & 1805 \\
\hline Moosbrunn & 1553 & 1349 & 872 & 1004 \\
\hline
\end{tabular}




\section{EXPERIMENTAL RESULTS}

In the measurement campaign, all HF radio signals were captured for 5-second duration with a one-minute interval between start times of two captures from the same transmitter. The sampling rate was set equal to $200 \mathrm{kHz}$. Using azimuthal equidistant projection system, the GPS coordinates of the HF transmitters and all receivers in the network were converted into a rectangular coordinate system. Coordinates of the selected reference receiver were used as the origin of the coordinate system. Details of the captures from different HF transmitters over several days are summarized in Table 2. Some of these captured data from different HF transmitters are analyzed and discussed in the following section.

Table 2 Details of capture schedule from different HF transmitters

\begin{tabular}{|c|c|c|c|c|}
\hline Date & $\begin{array}{c}\text { Transmitter } \\
\text { site }\end{array}$ & $\begin{array}{c}\text { Capture } \\
\text { freq (MHz) }\end{array}$ & $\begin{array}{c}\text { Capture } \\
\text { time (UTC) }\end{array}$ & $\begin{array}{c}\text { No. of } \\
\text { captures }\end{array}$ \\
\hline $17-07-17$ & Galbeni & 15.130 & $13: 15-13: 18$ & 4 \\
\hline $18-07-17$ & SMDG & 15.570 & $16: 11-16: 20$ & 10 \\
\hline $21-07-17$ & Nauen & 11.790 & $19: 11-19: 20$ & 10 \\
\hline $25-07-17$ & Cerrik & 13.665 & $11: 21-11: 25$ & 5 \\
\hline $27-07-17$ & Moosbrunn & 11.880 & $20: 11-20: 15$ & 5 \\
\hline
\end{tabular}

\section{A. Measurement Example: Favorable Propagation Environnent}

A HF radio signal which was captured synchronously by the four receivers on $21^{\text {st }}$ July at 19:17 UTC from Nauen is studied. The received signals on all receivers were demodulated and the message signal was extracted as explained in the earlier section. The signal received in Grenoble was considered as the reference signal on the basis of the received signal power. An example of propagation duration difference between the HF signal received in Grenoble and Brest is presented in Fig. 3, calculated by using cross-correlation technique. As seen in Fig. 3, there is a single cross-correlation peak which corresponds to one-hop propagation mode for signals received by both receivers. Using obtained TDoA estimates, the HF transmitter site was located with an error as small as $1.1 \mathrm{~km}$. This error corresponds to a relative error of about $0.1 \%$ calculated with respect to the maximum ground range among the four possible HF links. Fig. 5 presents the actual and estimated geographic location of the HF Tx in Nauen.

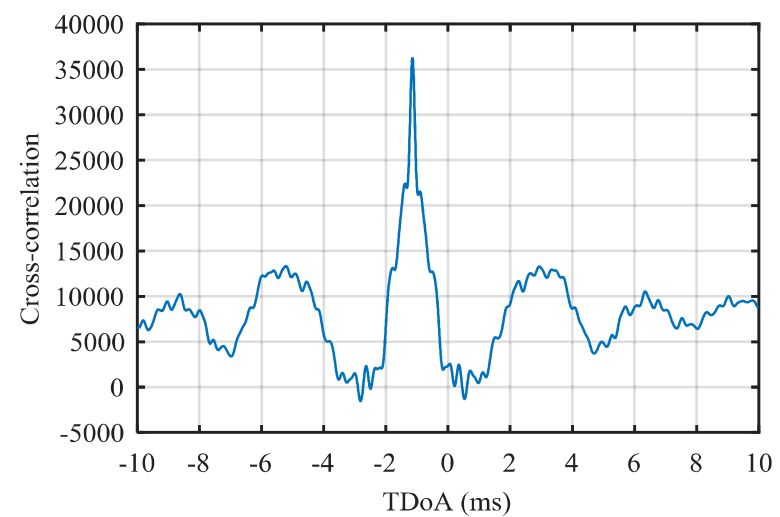

Fig. 3 TDoA estimate for HF signal captured in Grenoble and Brest from HF transmitter located in Nauen, Germany on 21st July, 2017 at 19:17 UTC

\section{B. Measurement Example: Moderate Geolocation Error}

This example provides the analysis of a HF signal captured from the HF transmitter located in SMDG by all receivers in the network on $18^{\text {th }}$ July at 16:11 UTC. Based on the received signal strengths at different receivers, the signal received in Grenoble was considered as the reference signal. Further, the received signals were post processed as explained earlier and three TDoA estimates were obtained between the signal received in Grenoble-Brest, Grenoble-Bordeaux and Grenoble-Lille, respectively. Using the geolocation algorithm, the Tx coordinates were estimated and the geolocation error was about $82 \mathrm{~km}$. The geolocation error seems moderate and is equivalent to a relative error of about $5.5 \%$. The actual and estimated transmitter location can be seen in Fig. 5.

\section{Measurement Example: Geometric Dilution of Precision}

In this example, data captured from Moosbrunn on $27^{\text {th }}$ July at 20:11 UTC are analyzed. The signal received in Grenoble is considered as the reference signal. Using the known receiver positions and the TDoA estimates, the geographic location of the transmitter is estimated as illustrated in Fig. 5. The geolocation error is large, about $303 \mathrm{~km}$ which corresponds to a relative error of $19.5 \%$. Geolocation based on TDoA estimations suffers from an error caused by long ranges from the receiver's baseline. The baseline refers to the distance between receivers. TDoA based systems locate the target by using multilateration principle at intersection of hyperbolas. For geolocation of the $\mathrm{Tx}$ in Moosbrunn and Galbeni using our receiver network, it is found that the hyperbolic line of positions (LOPs) are nearly parallel when exact TDoA values are known. A small error in TDoA due to noise results in large geolocation error and this is referred as Geometric Dilution of Precision (GDOP). The GDOP gets worse when the Tx's are farther away from receiver baselines [13], which can be seen from the geolocation errors obtained for the Tx in Galbeni. This example explains a case where a slight error in the estimated TDoA's leads to a large geolocation error.

\section{Measurement Example: Effect of Multipath Propagation}

This example investigates data captured from the HF transmitter located in Cerrik on $25^{\text {th }}$ July at 11:25 UTC. The signal received in Lille was considered as the reference signal considering the received signal intensity on different receivers. By cross-correlating the reference signal with the signal received by other receivers, estimates of TDoA's were obtained. Fig. 4 presents the cross-correlation output for the signals captured in Lille and Grenoble. As seen in Fig. 4, there are two peaks separated by $220 \mu \mathrm{sec}$ in cross-correlation output with different amplitudes. This implies that the signal was received at one of the receivers through multi-path channels. We have discussed the effect of peak selection on the geolocation error in [12]. In the present work, the time delay corresponding to the maximum power was selected as the TDoA estimate. Finally, Tx coordinates were estimated using the geolocation algorithm. The geolocation error was about $162 \mathrm{~km}$ corresponding to a relative error of about $8 \%$. The geographic location of estimated Tx can be seen in Fig 5. 


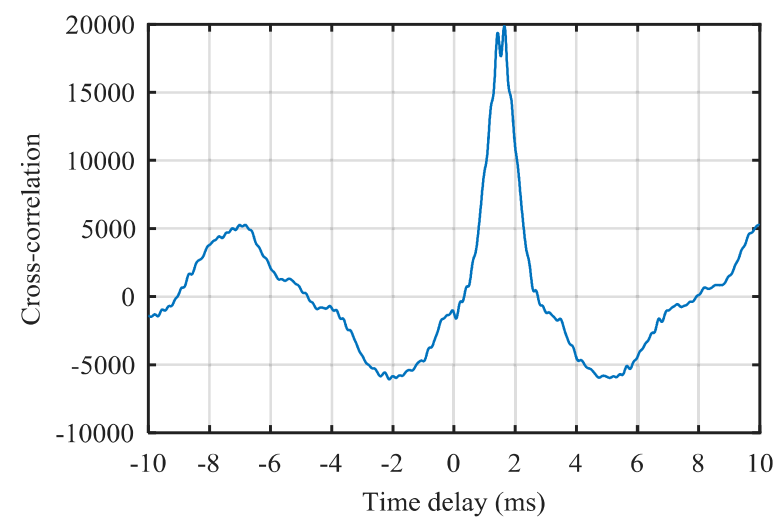

Fig. 4 TDoA estimate for HF signal captured in Lille and Grenoble from Cerrik, Albania on $25^{\text {th }}$ July at 11:25 UTC

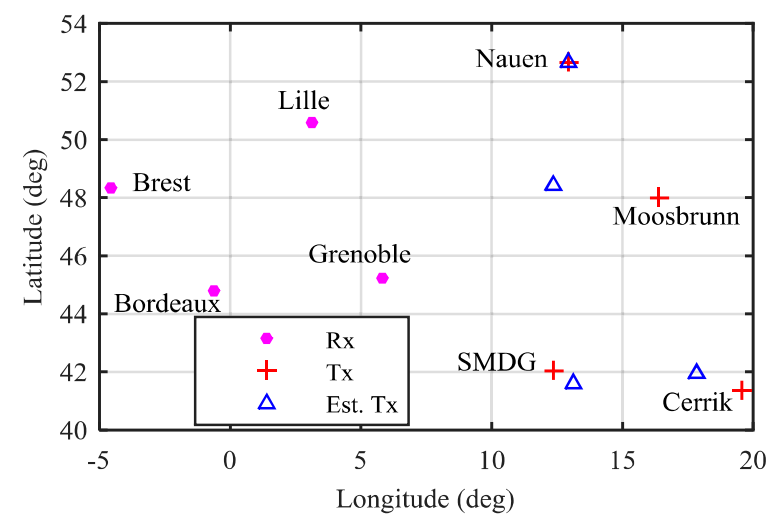

Fig. 5 Estimated and actual geographic location of HF transmitters for different measurement examples

Table 3 presents the summary of geolocation error for all the HF radio signals captured from different transmitters around Europe. Out of the five captures from Cerrik, only two captures were exploitable. For the other three captures, the data captured in Brest was too noisy thereby making them unusable. The error in terms of distance for the Tx located in Moosbrunn and Galbeni were found to be large mainly due to GDOP but the algorithm output in terms of direction of localization was approximately correct. Moreover, the geolocation algorithm assumes one-hop propagation mode and a uniform ionosphere which might not be the case for all captured signals. For all HF signals captured from SMDG and Nauen, the geolocation error was always less than $7 \%$.

Table 3 Geolocation error for data captured from different HF transmitters

\begin{tabular}{|c|c|c|c|}
\hline $\begin{array}{c}\text { Tx (Captures } \\
\text { considered) }\end{array}$ & $\begin{array}{c}\text { Error min. } \\
(\mathrm{km}, \boldsymbol{\%})\end{array}$ & $\begin{array}{c}\text { Error max. } \\
(\mathrm{km}, \boldsymbol{\%})\end{array}$ & $\begin{array}{c}\text { Error median } \\
(\mathrm{km}, \%)\end{array}$ \\
\hline Galbeni (3) & $39.96, \mathbf{1 . 6 8}$ & $845.88,35.57$ & $504.19,21.20$ \\
\hline SMDG (10) & $44.02, \mathbf{2 . 9 5}$ & $96.69,6.48$ & $82.28,5.51$ \\
\hline Nauen (10) & $1.16, \mathbf{0 . 0 8}$ & $71.94,5.44$ & $30.77,2.33$ \\
\hline Cerrik (2) & $162.13, \mathbf{7 . 9 0}$ & $283.14,13.81$ & - \\
\hline Moosbrunn (5) & $138.79, \mathbf{8 . 9 3}$ & $464.66,29.90$ & $303.15,19.51$ \\
\hline
\end{tabular}

\section{CONCLUSION}

In this letter, we presented first results of geolocation of $\mathrm{HF}$ sources which are located in the range of about $500-2500 \mathrm{~km}$ by using the TDoA method. It is explicitly assumed that signals follow one-hop propagation mode. In order to assess the feasibility of HF geolocation using the TDoA method, four single antenna receivers were deployed in four different cities in France. We performed a first series of experimental measurements with different HF stations located around Europe on different days. The TDoA estimates are obtained using the cross-correlation method. From the known receiver positions and estimated TDoA's, the Tx location is estimated using the geolocation algorithm. Geolocation performance is presented and discussed for HF signals captured from different transmitters at different times of different days.

In favorable conditions, the minimum geolocation error for all the HF Tx's considered in this study is less than $10 \%$. Considering the coverage area for one-hop HF propagation mode in $\mathrm{km}$, these errors are small and acceptable. Obtained geolocation errors can be further reduced by capturing data on a larger number of receivers [11]. From the analysis of geolocation results, it can be concluded that HF geolocation is possible using the TDoA method. In our future work, we plan to evaluate the confidence degree of an estimated location based on the output ionospheric height obtained from the geolocation algorithm or the level of received power at different receiving sites. Different super-resolution algorithm needs to be studied in order to reduce the quantization error of the TDoA estimates, which can further improve the geolocation accuracy.

\section{REFERENCES}

[1] K. Davies, "Ionospheric radio," IEE Electromagnetic Waves Series 31, pp. 124-152, n ISBN: 086341186 X, 1996.

[2] P. J. D. Gething, "HF direction finding," Proceedings of the Institution of Electrical Engineers, vol. 113, no. 1, pp. 49-61, 1966.

[3] R. O. Schmidt, "Multiple emitter location and signal parameter estimation", IEEE Trans. Antennas Propag., vol. AP-34, no. 3, pp. 276280, Mar 1986

[4] R. Roy, T. Kailath, "ESPRIT_Estimation of signal parameters via rotational invariance techniques", IEEE Trans. Acoust. Speech Signal Processing, vol. 37, no. 7, pp. 984-995, Jul 1989.

[5] Y. Zhang and Z. Ye, "Efficient Method of DOA Estimation for Uncorrelated and Coherent Signals," in IEEE Antennas and Wireless Propagation Letters, vol. 7, pp. 799-802, 2008.

[6] Y. Erhel, D. Lemur, L. Bertel and F. Marie, "H.F. radio direction finding operating on a heterogeneous array: principles and experimental validation," in Radio-Science, vol 39, n¹, pp. 1003:1-14, Jan-Feb 2004.

[7] G. Fabrizio and A. Heitmann, "A multipath-driven approach to HF geolocation," Signal Processing, vol. 93, no. 12, pp. 3487-3503, Dec. 2013.

[8] Y. Erhel and F. Marie, "An Operational HF System for Single Site Localization," in IEEE Military Communications Conference, 2007. MILCOM 2007, 2007, pp. 1-5.

[9] D. J. Torrieri, "Statistical theory of passive location systems," IEEE Trans. on Aerospace and Electronic Systems, vol. AES-20, no. 2, pp. 183-198, Mar. 1984.

[10] P. Pagani, R. Fleury, Y. L. Roux, and D. L. Jeune, "A study of HF transmitter geolocation through single-hop ionospheric propagation," The 8th European Conference on Antennas and Propagation (EuCAP), 2014, pp. 2689-2693.

[11] A. Jain, P. Pagani, R. Fleury, M. M. Ney, and P. Pajusco, "Efficient time domain HF geolocation using multiple distributed receivers," The 11th European Conference on Antennas and Propagation (EuCAP), 2017, pp. 1852-1856.

[12] A. Jain, P. Pagani, R. Fleury, M. M. Ney, and P. Pajusco, "CrossChannel Sounding for HF Geolocation: Concepts and Experimental Results," The 12th European Conference on Antennas and Propagation (EuCAP), 2018, accepted for publication.

[13] R. A. Poisel, "Electronic Warfare Target Location Methods," Second Edition, Artech House, pp. 226-252, n ISBN 13: 978-1-60807-523-2. 